\title{
RELEVANCIA DE LAS VARIABLES MOTIVACIONALES Y DE PERCEPCIÓN DE CONTROL EN EL PENSAMIENTO CREATIVO DE MUJERES Y HOMBRES DE LA POBLACIÓN GENERAL
}

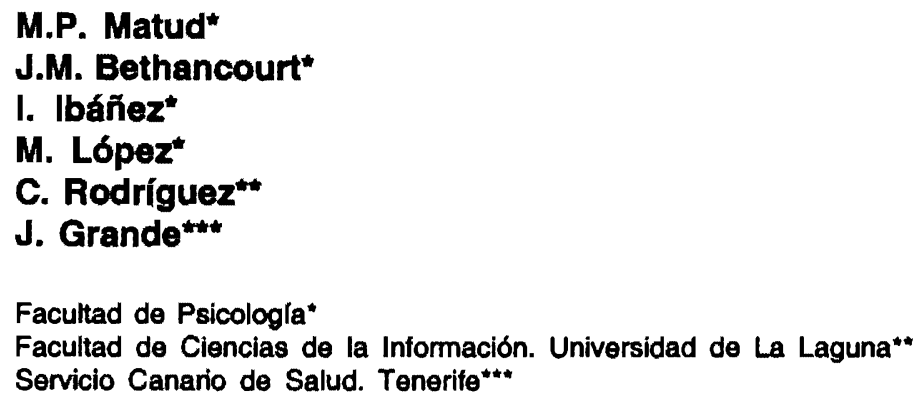

\section{RESUMEN}

Analizamos la relevancia de las variables motivacionales de rendimiento y de la percepción de control en el pensamiento creativo de una muestra de 180 hombres y 260 mujeres de la población general de edades comprendidas entre 18 y 65 años $(M=28,68, D T=10,38)$. La evaluación se realizó con las formas gráfica y verbal de los Test de Pensamiento Creativo de Torrance y con los cuestionarios de Motivación y Ansiedad de Ejecución y de Locus de Control de Pelechano. Aunque el porcentaje de varianza explicado fue bajo, los análisis de regresión mostraron que las variables más relevantes en el pensamiento creativo, tanto gráfico como verbal, de los

Correspondencia: M. Pilar Matud. Facultad de Psicología. Universidad de La Laguna. Campus de Guajara, 38205 La Laguna, Tenerife, España. E-mail: pmatud@ull.es.

Este trabajo forma parte del proyecto de investigación SEJ2004-00749/PSIC, subvencionado por la Dirección General de Investigación del Ministerio de Educación y Ciencia. 
hombres era la reacción de inhibición ante el estrés, mostrando mayor pensamiento creativo los hombres con menor reacción de inhibición ante las situaciones de estrés. Además, en dicho género, la motivación positiva hacia la acción se asoció con la puntuación en creatividad gráfica y la autoexigencia laboral con creatividad verbal. En el grupo de mujeres encontramos que puntuaban más alto en creatividad verbal las que mostraban menor indiferencia laboral y separación entre el mundo privado y laboral, mientras que mayor creatividad gráfica se asociaba con una menor percepción de control externo de resultados de acciones con componentes depresivos y miedo a la novedad.

Palabras clave: PENSAMIENTO CREATIVO, MOTIVACIÓN, LOCUS DE CONTROL, GÉNERO.

\section{SUMMARY}

This study analyzes the relevance of motivational achievement and locus of control variables on creative thinking. The sample was comprised of 180 men and 260 women from the general population, between 18 and 65 years old $(M=28.68$; $S D=10.38$ ). All sample members were assessed with the figural and verbal forms of the Torrance Test of Creative Thinking (TTCT) and with Pelechano's questionnaires on Motivation, Achievement Anxiety and Locus of Control. Although the percentage of variance explained was low, regression analyses showed that the most important variables in men's creative thinking, both figural and verbal, was the reaction of inhibition against stress; men with less reaction of inhibition against stress showed greater creative thought. In addition, positive motivation to action was associated with figural creativity, while work selfdemanding was associated with verbal creativity. In the women's group, those with higher scores on verbal creativity showed less work indifference and less separation between work and private space. Among the women, figural creativity was associated with 
a perception of less external control over results of actions with depressive components and fear of novelty.

Key wordS: SOCIAL ANXIETY DISORDER, PSYCHOPATHOLOGIES, COMPARISON OF GROUPS, ADOLESCENTS, CLINICAL INDEXES.

\section{INTRODUCCIÓN Y OBJETIVO}

Como señala Simonton (2000), la creatividad es, de todas las actividades humanas, una de las más omnipresente e importante y se ha considerado como un atributo positivo para la persona que lo posee, si bien pocos psicólogos la han considerado como un tópico central de investigación. El estudio de las relaciones entre creatividad y personalidad se ha realizado durante varias décadas por diversos autores, existiendo varios intentos de describir la «personalidad creativa» (Burch, Hemsley, Pavelis y Corr, 2006). Como señalan Tardif y Sternberg (1988), la descripción típica de la persona creativa suele incluir tres aspectos: las características cognitivas, las de personalidad y la motivación. Welsh (1975), tras revisar el trabajo de varios autores, plantea que la persona creativa se caracteriza por los siguientes rasgos: 1) independencia, tanto en sus actitudes como en su conducta social, 2) dominancia, 3) introversión, 4) apertura ante estímulos, 5) amplitud de intereses, 6) aceptación de sí misma, 7) intuición, 8) flexibilidad, 9) presencia y equilibrio social, 10) actitud antisocial, y 11) despreocupación por las normas sociales. Señala, además, dos rasgos adicionales que parecen estar más asociados al arte que a la creatividad científica: el radicalismo y el rechazo de las constricciones sociales. Sternberg (1988) plantea la existencia de tres facetas en la creatividad, dos que tienen que ver con los aspectos intelectuales y en la forma en que son aplicados a través de los estilos cognitivos y una tercera que tiene que ver con la personalidad. Aunque reconoce que no se trata de una lista exhaustiva ni que todas las personas creativas tienen todas las características citadas, reconoce la importancia en la creatividad de ciertas características de personalidad tales como la tolerancia a la ambigüedad, la persistencia para superar obstáculos, el deseo de crecer, la motivación intrínseca, la toma moderada de riesgos, el deseo de reconocimiento y el trabajo para 
lograr tal reconocimiento. En un trabajo posterior (Sternberg, 2006a) afirma que la creatividad requiere la confluencia de seis recursos distintos pero interrelacionados: las capacidades intelectuales, el conocimiento, los estilos de pensamiento, la personalidad, la motivación y el medio que apoya y reconoce las ideas creativas.

Csikszentmihalyi (1996) considera que la creatividad es fruto de la interacción entre los pensamientos de una persona y un contexto sociocultural, siendo más un sistema que un fenómeno individual. Plantea que la persona creativa se caracteriza por la complejidad, siendo capaz de alternar entre polos opuestos de características si la ocasión así lo requiere, siendo capaz de adaptarse a las condiciones particulares de un determinado campo, las cuales son diferentes en función del tiempo y del campo de creatividad de que se trate.

Eysenck (1995) plantea que la creatividad es una capacidad o rasgo disposicional que permite a la persona conjugar ideas o ejecutar y producir trabajos imaginativos, que tienen una apariencia de novedad y que son aceptados por expertos y colegas como contribuciones genuinas con valor social. Pero poseer tal rasgo no garantiza el logro creativo, siendo necesarias otras condiciones y otros rasgos, tales como fuerza del yo, variables motivacionales y cognitivas, junto con variables socioculturales.

Se han planteado diversos factores motivacionales implicados en la creatividad, aunque tampoco existe unanimidad al respecto. Welsh (1975) afirma que pueden diferenciarse dos perspectivas distintas: una de orientación negativa, que se ha ocupado sobre todo de la creación artística, que plantea que el origen de la creatividad está en impulsos oscuros e inaceptables y otra positiva que considera la creatividad como el resultado natural de la realización y expresión del desarrollo de los potenciales humanos. La mayor parte de los autores que han seguido la primera perspectiva provienen del campo psicoanalítico y han destacado la relevancia de caracteristicas tales como la agresión, hostilidad, impulsos destructivos, ansiedad, culpa, necesidad de restitución, sublimación..., entre otros conceptos psicoanalíticos. Aunque el autor más representativo de la segunda perspectiva es Carl Rogers (1954) que considera la persona creativa como motivada por la necesidad de auto realizarse y desarrollar todo su potencial, muchos otros autores se engloban dentro de esta 
perspectiva, que se caracteriza por mayor nigor experimental si bien el apoyo empírico obtenido es bastante dispar (Welsh, 1975). Según este autor, dentro de esta perspectiva la persona creativa se caracterizaría por una motivación seria, significativa y dirigida, más que casual, incidental y accidental.

También otros autores plantean la relevancia en la creatividad del tipo de motivación, destacando el papel de la motivación intrínseca (véase, por ejemplo, Down, 1989; Sternberg, 2006b). Amabile (1983) plantea que, mientras la motivación intrínseca aumenta la creatividad, la motivación extrínseca parece reducirla, efectos que parecen darse tanto en la infancia como en las personas adultas (Amabile, Hennessey y Grossman, 1986). Pero tampoco existe acuerdo en los efectos que tiene el refuerzo sobre la creatividad, ya que se ha encontrado que el reforzar la ejecución novedosa aumenta la motivación intrínseca y la creatividad mientras que el refuerzo de la ejecución tradicional disminuye ambos fenómenos (Eisenberguer y Shanock, 2003). Y también parecen existir algunas diferencias de género en los efectos de la motivación extrínseca sobre la creatividad, habiéndose encontrado que la expectativa de ser valorado y el hacer algo para obtener recompensa disminuía la creatividad de las chicas pero no la de los chicos (Baer, 1997, 1998).

El Locus de control se refiere a la medida en que las personas perciben que están controladas intemamente, es decir, por sus propios esfuerzos o capacidades, o externamente, es decir, por fuerzas ambientales tales como la suerte o las acciones de otras personas (Dowd, 1989). Aunque las primeras formulaciones del locus de control plantearon la existencia de una expectativa de control generalizado posteriormente se reconoció la multidimensionalidad del constructo. Pelechano (2000) conceptualiza el locus de control como la expectativa acerca de la responsabilidad en el control de las acciones y sus consecuencias $y$, en estudios realizados con personas adultas, encontró varios factores de primer orden que se agrupaban en tres factores de segundo orden.

Se ha considerado que las personas con locus de control interno serán más creativas que las que tienen un locus de control externo, ya que se cree que serán más capaces de implicarse en la rumiación cognitiva precisa para la creatividad (Down, 1989). También se ha 
planteado que las personas con locus de control interno tienen muchos de los atributos asociados a la creatividad, tales como autonomía, búsqueda de información para el cambio, independencia o autoconfianza (Strickland, 1989). Y existe evidencia empírica de que, mientras que las personas con locus de control interno tienden a asumir el control, las que lo tienen externo toman roles más pasivos y confían menos en sí mismas (Bolen y Torrance, 1978). Pero aunque se han encontrado asociaciones entre locus de control interno y algunas medidas de pensamiento creativo, tales como fluidez 0 flexibilidad, la evidencia empírica no es completa (véase, por ejemplo, Glover y Sautter, 1976; Bolen y Torrance, 1978).

El objetivo del presente trabajo es analizar la relevancia de las variables motivacionales de rendimiento y de la percepción de control en el pensamiento creativo de las mujeres y los hombres adultos de la población general.

\section{MÉTODO}

\section{Sujetos}

La muestra está formada por un total de 440 personas de la población general que participaron voluntariamente en el estudio. Sus edades estaban comprendidas entre 18 y 65 años, siendo la edad media de 28,68 y la desviación típica de 10,38 . Algo más de la mitad (el $59,1 \%$ ) son mujeres y el $40,9 \%$ son hombres. En la tabla 1 presentamos los principales datos sociodemográficos. Como puede observarse, se da bastante diversidad en el nivel de estudios y laboral, así como en el estado civil, si bien es mucho más común que las personas estén solteras y es poco frecuente que estén divorciadas separadas o viudas. No hay diferencias estadisticamente significativas entre mujeres y hombres en las variables sociodemográficas, excepto en el nivel laboral.

\section{Instrumentos}

Tests de Pensamiento Creativo (TTCT, Torrance, 1990a, 1990b). Están formados por dos baterias de tests, una gráfica y otra verbal de 
Relevancia de las variables motivacionales...

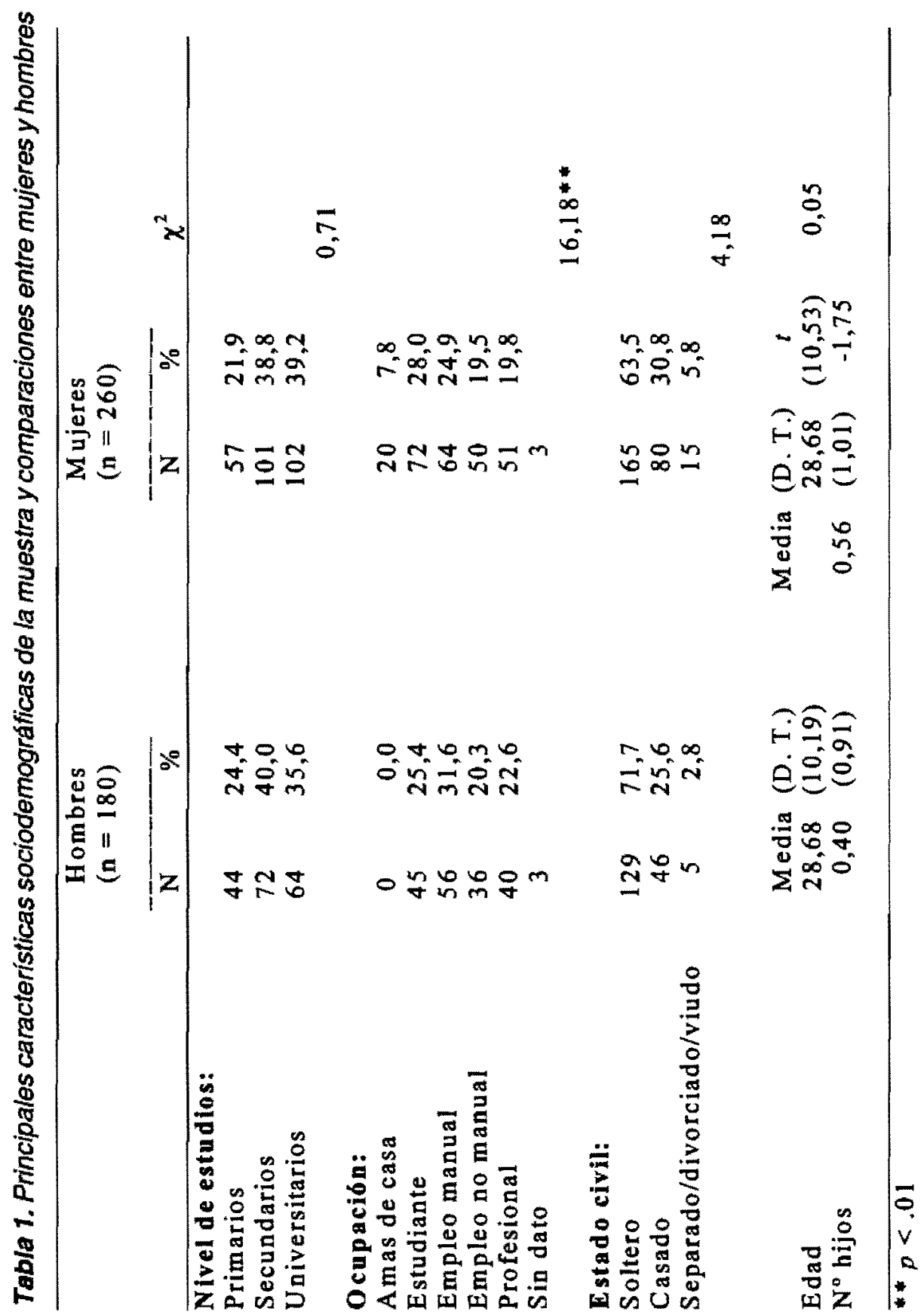


las cuales existen formas paralelas. La gráfica consiste en 3 actividades a cumplimentar en 10 minutos cada una y permite obtener 5 puntuaciones normativas (fluidez, originalidad, abstracción de títulos, elaboración y resistencia al cierre prematuro) y 13 medidas referidas a un criterio (expresividad emocional, articulación de la historia, movimiento 0 acción, expresividad de los títulos, síntesis de figuras incompletas, síntesis de líneas o de círculos, visualización inusual, visualización interna, ruptura de límites, humor, riqueza de la imagen, colorido de la imagen, y fantasía) que, sumadas a las puntuaciones típicas medias de las puntuaciones normativas, permiten obtener un indice de creatividad, que es un indicador global del potencial creativo (Torrance, 1990a).

La forma verbal consiste en 6 actividades, que se realizan en 50 10 minutos cada una, siendo el tiempo total de 40 minutos. Con todas ellas se obtienen tres tipos de puntuaciones: fluidez, flexibilidad y originalidad, cuyas puntuaciones típicas se promedian para obtener una puntuación media que es un indicador global de la fuerza creativa (Torrance, 1990b).

EI TTCT ha sido traducido a más de 35 idiomas (Millar, 2002) y su uso ha sido recomendado no solo en el área educativa sino también en la laboral (Kim, 2006), siendo la prueba que más se ha utilizado para evaluar el talento creativo (Sternberg, 2006a). Los estudios sobre la fiabilidad de las puntuaciones del TTCT han mostrado que puede ser superior a .90 (Torrance, 1990a) y los estudios de validez han mostrado evidencia de la validez de predictiva y de contenido del TTCT (Kerr y Gagliardi, 2003; Kim, 2006; Plucker, 1999; Torrance, 1981; Torrance y Ball, 1984; Wechsler, 2006).

Motivación y ansiedad de ejecución (MAE, Pelechano, 1975). Está compuesto por 72 ítems con dos alternativas de respuesta (si / no) que se agrupan en 6 factores, cuatro de motivación y dos de ansiedad: tendencia a la sobrecarga de trabajo, indiferencia laboral y separación entre el mundo privado y el laboral, autoexigencia en el trabajo, motivación general positiva hacia la acción, reacción de inhibición ante situaciones de estrés, y reacción de activación y acción en situaciones de estrés.

Cuestionaio de Locus de Control (LUCAM, Pelechano y Báguena, 1984). Aunque el cuestionario original estaba formado por 87 ítems 
con escala de respuesta de cuatro puntos (nunca, alguna vez, frecuentemente, siempre), en estudios de validación posteriores se redujo a 62 ítems (Pelechano, 2003, tomado de Pastor y Pelechano, 2006). La estructura factorial en la población general mostró la existencia de tres factores de segundo orden: 1) control externo en resultados de acciones con componentes depresivos y miedo a la novedad; 2) control bipolar de exculpación en tareas de grupo y exigencia personal en el logro de metas; 3) control externo social generalizado sobre logros en el trabajo y resultado de éxito o fracaso en las relaciones personales.

\section{Procedimiento}

El pase de pruebas de los tests de creatividad fue realizado de forma grupal por personal entrenado y siguiendo las intrucciones de los manuales del TTCT (Torrance, 1990a, 1990b). En el caso del alumnado, las pruebas de personalidad también fueron realizadas de forma grupal y en la muestra de la población general fue autoadministrado o en forma de entrevista estructurada en función del nivel de estudios. El acceso a la muestra de estudiantes fue a través de centros educativos y la muestra de la población general se obtuvo a partir de personas conocidas de un grupo de alumnos de psicología que, una vez entrenados, participaron en el pase de pruebas. Los análisis estadísticos se han realizado con la versión 12 del programa SPSS para Windows.

\section{RESULTADOS}

En la tabla 2 se muestra los coeficientes de correlación de Pearson entre las diez medidas de pensamiento creativo y los factores de motivación de rendimiento y locus de control en la muestra de hombres. Como puede observarse, aunque hay algunos coeficientes de correlación estadísticamente significativos, la magnitud de la asociación es baja en todos los casos. De los seis factores del cuestionario de motivación de rendimiento, el que más se asocia con el pensamiento creativo es el factor de reacción de inhibición ante estrés, que correlaciona de forma negativa con fluidez, tanto gráfica 
como verbal, con originalidad verbal, con el índice de creatividad y con la media verbal. El factor de indiferencia laboral y separación entre mundo privado y laboral correlaciona de forma negativa con la resistencia al cierre prematuro; el de motivación positiva hacia la acción y la reacción de facilitación ante situaciones de estrés correlacionan de forma positiva con el índice de creatividad, mientras que el factor de control externo en resultados de acciones con componentes depresivos y de miedo a la novedad lo hace negativamente.

Las correlaciones entre las medidas de pensamiento creativo y los factores de motivación de rendimiento y locus de control en la muestra de mujeres se muestran en la tabla 3 . Se observa que, aunque hay un mayor número de coeficientes de correlación estadísticamente significativos que en la muestra de hombres, su magnitud es baja en todos los casos. Los factores de indiferencia laboral y separación entre el mundo privado y laboral y de control externo en resultados de acciones con componentes depresivos y de miedo a la novedad se asocian negativamente con todas las medidas de creatividad verbal excepto flexibilidad; con abstracción de títulos y con el índice de creatividad. El factor de motivación positiva hacia la acción correlaciona positivamente con fluidez verbal y con la media de creatividad verbal y negativamente con originalidad gráfica; la tendencia a la sobrecarga de trabajo correlaciona negativamente con elaboración, la reacción de inhibición ante el estrés correlaciona negativamente con fluidez gráfica y el control externo social generalizado correlaciona positivamente con la resistencia al cierre prematuro.

Dado que tanto las diversas medidas de pensamiento creativo como algunos de los factores de motivación de rendimiento y de locus de control correlacionaban entre sí, se realizaron análisis de regresión múltiple para conocer qué variables de motivación y de percepción de control eran los mejores predictores de los índices globales de creatividad. El modelo de regresión utilizado fue el paso a paso, introduciendo en la ecuación de regresión como variables independientes los seis factores de motivación y los tres de locus de control y siendo el criterio a predecir el índice de creatividad gráfico en un análisis y la puntuación media verbal en el otro. Ambos análisis se realizaron de forma independiente para la muestra de mujeres y para la de hombres. 


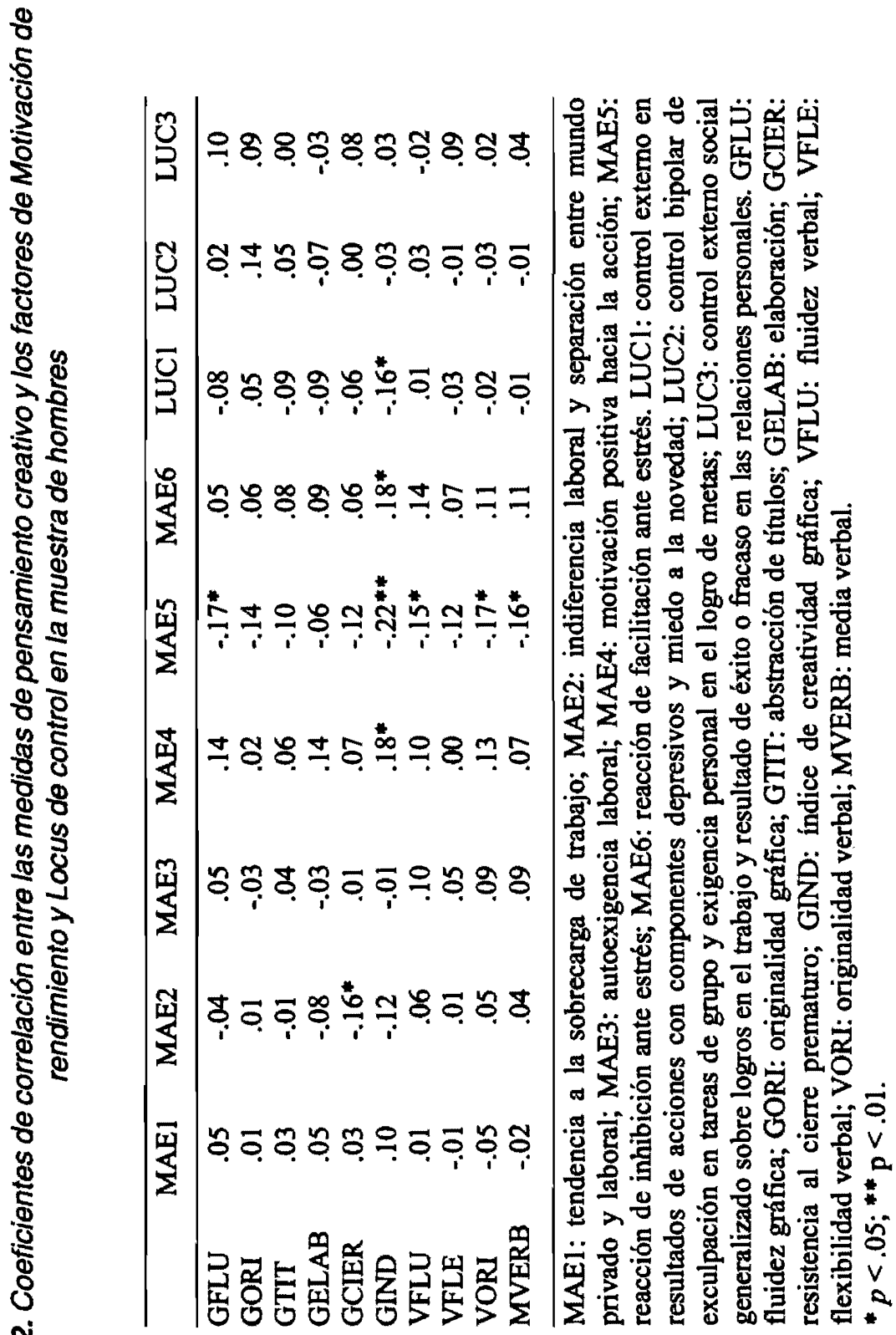




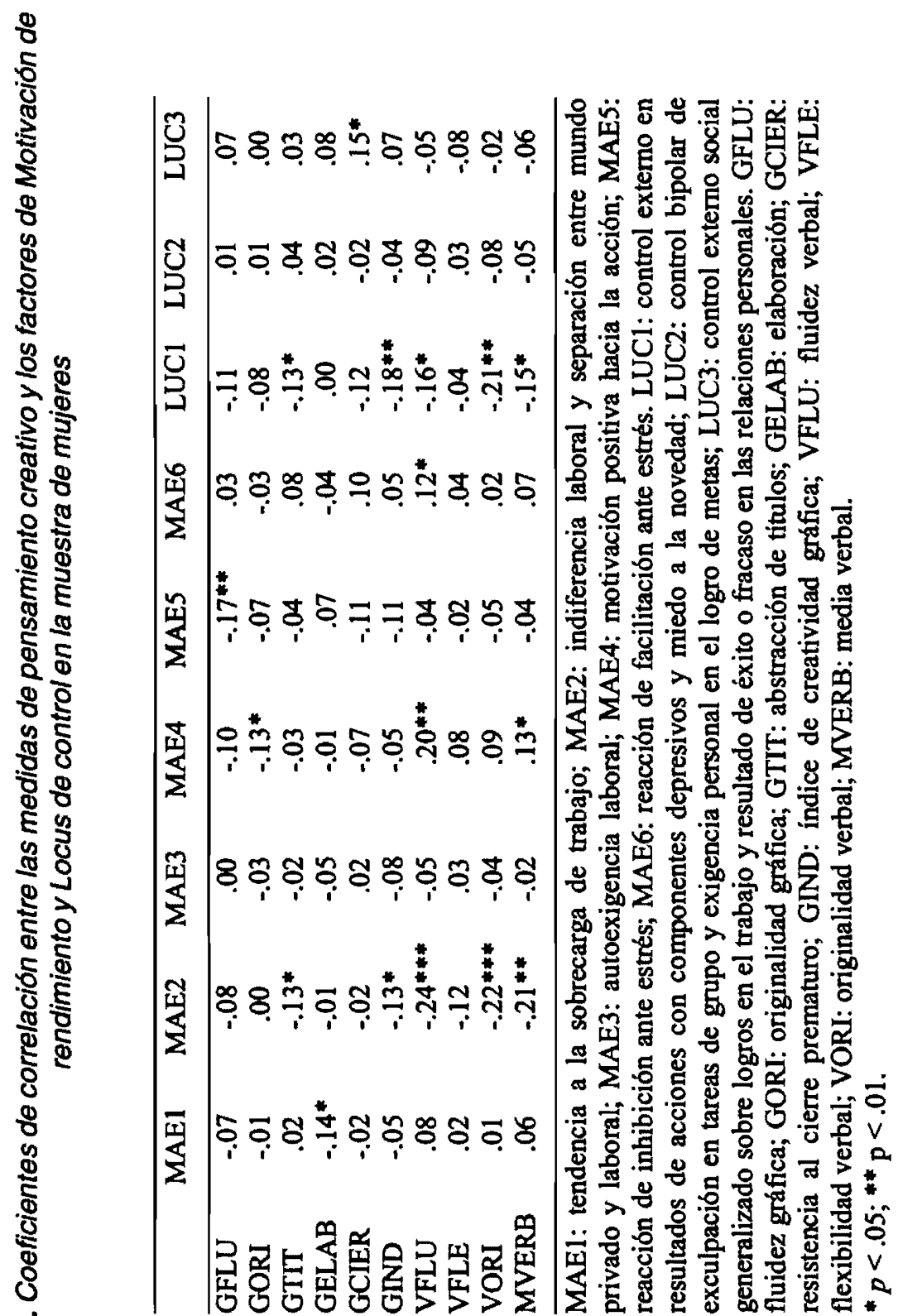


En la muestra de hombres, al predecir el índice gráfico de creatividad encontramos que solo entraron en la ecuación de regresión dos factores: el de reacción de inhibición ante el estrés (MAE5) y el de motivación positiva ante la acción (MAE4). Como puede observarse en la tabla 4, el peso Beta del factor MAE5 es negativo y su magnitud es ligeramente superior a la del MAE4.

Tabla 4. Análisis de regresión paso a paso prediciendo el índice gráfico de creatividad en la muestra de hombres

\begin{tabular}{lllllllll}
\hline Modelo & $\mathrm{R}$ & $\mathrm{R}^{2}$ & \multicolumn{3}{c}{$\mathrm{R}^{2}$ corregida $p$ cambio en F } & \multicolumn{3}{c}{ MODELO FINAL } \\
& & & & & Beta & $t$ & $p$ \\
\hline 1 & & .215 & .046 & .041 & .004 & & & \\
2 & & .290 & .084 & .074 & .007 & & & \\
2 & MAE5 & & & & & -.225 & -3.12 & .002 \\
2 & MAE4 & & & & & .195 & 2.70 & .007 \\
\hline
\end{tabular}

MAE4: Motivación positiva hacia la acción; MAE5: Reacción de inhibición ante estrés.

Al predecir la puntuación media de creatividad verbal en la muestra de hombres también se encontró que formaban parte de la ecuación de regresión dos factores: el de reacción de inhibición ante el estrés (MAE5), cuyo peso Beta era negativo, y el de autoexigencia laboral (MAE3) que tiene un peso Beta positivo y cuya magnitud es algo menor que la del MAE5 (véase tabla 5).

En la muestra de mujeres, al predecir el índice gráfico de creatividad, encontramos que solo un factor formó parte de la ecuación de regresión: el factor de control externo en resultados de acciones con componentes depresivos y miedo a la novedad (LUC1). Los estadísticos de este factor fueron $R=.178 ; R^{2}=.032$ y $R^{2}$ corregida $=$ .028 . El coeficiente Beta fue de $-.178(t=-2.91, p=.004)$. En la predicción de la puntuación media de creatividad verbal también entró a formar parte de la ecuación de regresión un solo factor: la indiferencia laboral y separación entre el mundo privado y laboral (MAE2). Los estadísticos fueron $R=.207 ; R^{2}=.043$ y $R^{2}$ corregida $=.039$. El coeficiente Beta fue de $-.207(t=-3.39, p=.001)$.

En la muestra de mujeres, al predecir el índice gráfico de creatividad, encontramos que solo un factor formó parte de la ecuación de regresión: el factor de control externo en resultados de acciones con 
656 M. P. Matud, J.M. Bethancourt, I. Ibáñez, M. López, C. Rodniguez, J. Grande

Tabla 5. Análisis de regresión paso a paso prediciendo la puntuación media en creatividad verbal en la muestra de hombres

\begin{tabular}{|c|c|c|c|c|c|c|c|}
\hline \multirow[t]{2}{*}{ Modelo } & \multirow[t]{2}{*}{$\overline{\mathbf{R}}$} & \multirow[t]{2}{*}{$\mathrm{R}^{2}$} & \multirow[t]{2}{*}{$\mathrm{R}^{2}$ corregida } & \multirow[t]{2}{*}{$p$ cambio en $\mathrm{F}$} & \multirow[b]{2}{*}{ Beta } & \multicolumn{2}{|c|}{ MODELO FINAL } \\
\hline & & & & & & $t$ & $p$ \\
\hline 1 & .160 & .025 & .020 & .032 & & & \\
\hline 2 & .220 & .048 & .037 & .041 & & & \\
\hline 2 MAE5 & & & & & -.209 & -2.71 & .007 \\
\hline 2 MAE3 & & & & & .159 & 2.06 & .041 \\
\hline
\end{tabular}

MAE3: Autoexigencia laboral; MAEs: Reacción de inhibición ante estrés.

componentes depresivos y miedo a la novedad (LUC1). Los estadísticos de este factor fueron $R=.178 ; R^{2}=.032$ y $R^{2}$ corregida $=$ .028. El coeficiente Beta fue de $-.178(t=-2.91, p=.004)$. En la predicción de la puntuación media de creatividad verbal también entró a formar parte de la ecuación de regresión un solo factor: la indiferencia laboral y separación entre el mundo privado y laboral (MAE2). Los estadísticos fueron $R=.207 ; R^{2}=.043$ y $R^{2}$ corregida $=.039$. El coeficiente Beta fue de $-.207(t=-3.39, p=.001)$

\section{CONCLUSIONES Y DISCUSIÓN}

En este trabajo hemos analizado la relevancia de las variables motivacionales de rendimiento y del locus de control en el pensamiento creativo de una muestra de mujeres y hombres adultos de la población general. Hemos encontrado que, pese a que el porcentaje de varianza común no alcanza el $10 \%$ en ninguna de las correlaciones, sí se dan algunas asociaciones estadísticamente significativas, difiriendo la mayor parte en función del género. Aunque en ambos géneros la flexibilidad verbal aparece como independiente de la motivación de rendimiento y del locus de control, el resto de las medidas de pensamiento creativo evaluadas por los TTCT de Torrance (1990a, $1990 \mathrm{~b})$ se asocian en uno u en otro género con alguno de tales factores.

Para profundizar en el conocimiento de cuáles eran las variables más relevantes en el pensamiento creativo de mujeres y hombres realizamos análisis de regresión múltiple $y$, aunque el porcentaje de varianza explicado era muy bajo, sí encontramos factores que predecían significativamente el rendimiento creativo global, tanto gráfico 
como verbal, de mujeres y hombres. Las variables más relevantes en el potencial creativo de los hombres, tal y como es evaluado por el TTCT gráfico de Torrance (1990a), eran la reacción de inhibición ante el estrés y la motivación positiva hacia la acción, caracterizándose los hombres con mayor potencial creativo por una menor reacción de inhibición ante el estrés y una mayor motivación positiva hacia la acción. En el caso de las mujeres, el porcentaje de varianza explicada fue menor y la variable relevante en el potencial creativo era el control externo en resultados de acciones con componentes depresivos y miedo a la novedad, mostrando menor potencial creativo las mujeres con mayor percepción de control externo de factores tales como hados y suerte en los resultados de acciones, y con componentes depresivos y miedo a la novedad. Estos resultados coinciden en alguna medida con los de otros autores que encuentran asociación entre locus de control interno y algunos indicadores de creatividad en las muestras de mujeres pero no en las de hombres (Brecher y Denmark, 1969).

A la hora de predecir la puntuación media en el TTCT verbal que es un indicador global de la fuerza creativa (Torrance, 1990b), la variables relevantes en el caso de los hombres eran la reacción de inhibición ante situaciones de estrés y la autoexigencia laboral. Según los resultados del presente estudio, los hombres con más fuerza creativa parecen ser los que tienen una menor reacción de inhibición ante el estrés y los que más se exigen a nivel laboral. En el caso de las mujeres, el mejor predictor de la fuerza creativa era el factor de indiferencia laboral y separación entre mundo privado y laboral, mostrando menor fuerza creativa las mujeres con mayor indiferencia laboral y mayor separación entre los mundos privado y laboral.

Respecto a las asociaciones entre medidas específicas del pensamiento creativo y los factores de motivación de rendimiento y locus de control comunes a mujeres y hombres, hemos encontrado que las personas con mayor reacción de inhibición ante el estrés tienden a presentar menor fluidez gráfica.

Pero aunque tales resultados son muy sugerentes e indican que las variables motivacionales y de percepción de control parecen tener una cierta relevancia diferencial en el pensamiento creativo de mujeres y hombres, no hay que olvidar que el porcentaje de varianza explicada es muy baja. Otra limitación del presente estudio es que, dado que se 
trata de un estudio transversal no puede hablarse de predicción, sino solo de asociación entre variables. Además, dado que la muestra no es aleatoria, los resultados no pueden ser generalizados a toda la población.

\section{REFERENCIAS BIBLIOGRÁFICAS}

Amabile, T. M. (1983). Motivation and creativity: Effects of motivational orientation on creative writers. Journal of Personality and Social Psychology, 48, 303-399.

Amabile, T. M., Hennessey, B. A. y Grossman, B. S. (1986). Social influences on creativity: The effects of contracted-for reward. Joumal of Personality and Social Psychology, 50, 14-23.

Baer, J. (1997). Gender differences in the effects of anticipated evaluation on creativity. Creativity Research Journal, 10, 25-31.

Baer, J. (1998). Gender differences in the effects of extrinsic motivation on creativity. Joumal of Creative Behavior, 32, 18-37.

Bolen, L. M. y Torrance, E. P. (1978). The influence on creative thinking of locus of control, cooperation and sex. Journal of Clinical Psychology, 34, 903-907.

Burch, G. J., Hemsley, D. R., Pavelis, C. y Corr, P. J. (2006). Personality, creativity and latent inhibition. European Journal of Personality, 20, 107-122.

Brecher, M. y Denmark, F. L. (1969). Internal-external locus of control and verbal fluency. Psychological Reports, 25, 707-710.

Csikszentmihalyi, M. (1996). Creativity. Flow and the psychology of discovery and invention. Nueva York: HaperPerennial.

Dowd, E. T. (1989). The self and creativity. Several constructs in search of a theory. En J. A. Glover, R. R. Ronning y C. R. Reynolds (Eds.) Handbook of Creativity. Nueva York: Plenum Press.

Eisenberguer, R. y Shanock, L. (2003). Rewards, intrinsic motivation, and creativity: A case study of conceptual and methodological isolation. Creativity Research Journal, 15, 121-130.

Eysenck, H. J. (1995). Genius. The natural history of creativity. Cambridge: Cambridge University Press.

Glover, J. A. y Sautter, F. (1976). An investigation of the relationship of four components of creativity to locus of control. Social Behavior and Personality, 4, 257-260. 
Kerr, B. y Gagliardi, C. (2003). Measuring creativity in research and practice. In S. J. Lopez y C. R. Snyder (Eds.) Positive psychological assessment: $A$ handbook of models and measures. Washington: American Psychological Association.

Kim, K. H. (2006). Can we trust creativity tests? Torrance Tests of Creative Thinking (TTCT). Creativity Research Journal, 18, 3-14. Millar, G W. (2002). The Torrance Kids at mid-life. Westport: Ablex. Pastor, A. y Pelechano, V. (2006). Lógica y resultados de un modelo predictivo de los trastornos de personalidad a partir de dimensiones de personalidad en distinto nivel de consolidación. Análisis y Modificación de Conducta, 32, 165-193.

Pelechano, V. (2000). Psicología sistemática de la Personalidad. Barcelona: Ariel.

Pelechano, V. (1975). El cuestionario MAE (Motivación y ansiedad de ejecución). Madrid: Fraser.

Pelechano, V. y Baguena, M. J. (1984). Un cuestionario de Locus de Control (LUCAM). Análisis y Modificación de Conducta, 20, 5-46.

Plucker, J. A. (1999). Is the proof in the pudding? Reanalyses of Torrance's (1958 to present) longitudinal data. Creativity Research Joumal, 12, 103-114.

Roger, C. R. (1954). Toward a theory of creativity. ETC: A Review of General Semantics, 11, 249-260.

Sternberg, R. J. (1988). A three-facet model of creativity. En R. J. Sternberg (Ed.) The nature of creativity. Contemporary psychological perspectives. Cambridge: Cambridge University Press.

Sternberg, R. J. (2006a). The nature of creativity. Creativity Research Journal, 18, 87-98.

Sternberg, R. J. (2006b). Creating a vision of creativity: The first 25 years. Psychology of Aesthetics, Creativity, and the Arts, 5, 2-12.

Simonton, K. D. (2000). Creativity. Cognitive, personal, developmental, and social aspects. American Psychologist, 55, 151-158.

Strickland, B. R. (1989). Internal-external control expectancies. From contingency to creativity. American Psychologist, 44, 1-12.

Tardif, T. Z. y Sternberg, R. J. (1988). What do we know about creativity? En R. J. Sternberg (Ed.) The nature of creativity. Contemporary psychological perspectives. Cambridge: Cambridge University Press. 
Torrance, E. P. (1981). Empirical validation of criterion-referenced indicators of creative ability through a longitudinal study. The Creative Child and Adult Quarterly, 3, 136-140.

Torrance, E. P. (1990a). Torrance Test of Creative Thinking. Normstechnical manual Figural (Streamlined) Forms A \& B. Benseville: Scholastic Testing Service Inc.

Torrance, E. P. (1990b). Manual for scoring and interpreting results.

Torrance Test of Creative Thinking. Verbal, Forms $A$ and $B$. Benseville: Scholastic Testing Service Inc.

Torrance, E. P. y Ball, O. E. (1984). Torrance Test of Creative Thinking. Streamlined (revised) manual. Figural $A$ and $B$. Benseville: Scholastic Testing Service Inc.

Welsh, G. S. (1975). Creativity and intelligence: A personality approach. Chapel Hill: Institute for Research in Social Science. University of North Carolina.

Wechsler, S. (2006). Validity of the Torrance Tests of Creative Thinking to the Brazilian Culture. Creativity Research Journal, 18, 15-25. 